The n-Hosoya Polynomials of the Composite of Some Special Graphs

\author{
Ahmed M. Ali \\ ahmedgraph@uomosul.edu.iq \\ College of Computer Sciences and Mathematics \\ University of Mosul, Mosul, Iraq
}

Received on: 03/05/2011

Accepted on: 21/06/2011

\begin{abstract}
It is not easy to find the n-Hosoya polynomial of the compound graphs constructed in the form $G_{1} \otimes G_{2}$ for any two disjoint connected graphs $G_{1}$ and $\mathrm{G}_{2}$. Therefore, in this paper, we obtain n-Hosoya polynomial of $G_{1} \otimes G_{2}$ when $G_{1}$ is a complete graph and $G_{2}$ is a special graph such as a complete graph, a bipartite complete, a wheel, or a cycle. The n-Wiener index of each such composite graph is also obtained in this paper.

Keywords: Composite graphs $G_{1} \otimes G_{2}, n$-Hosoya polynomial ,n-Wiener index.

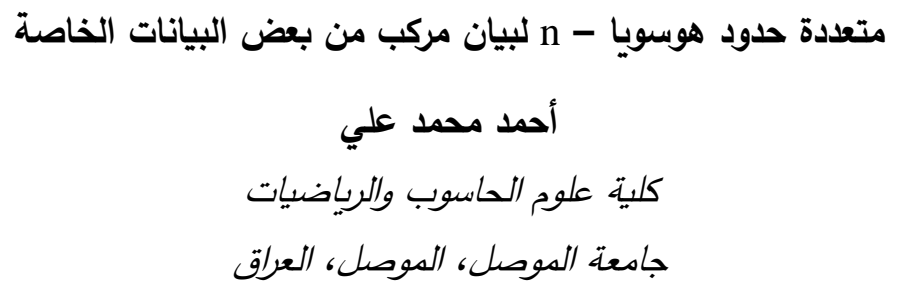

تاريخ قبول البحث: 2011/06/21

تاريخ استلام البحث: 2011/05/03

\section{الملخص}

إن إيجاد صيغة بسيطة لمتعددة حدود هوسويا-n لبيان مركب من بيانين متصلين G و G ومنفصلين

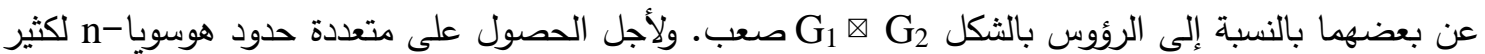

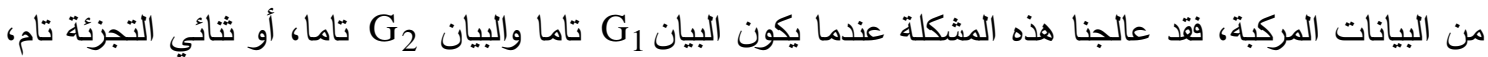
أو عجلة، أو دارة. ولقد أوجدنا دليل وينر -n لكل من البيانات المركبة المذكورة.

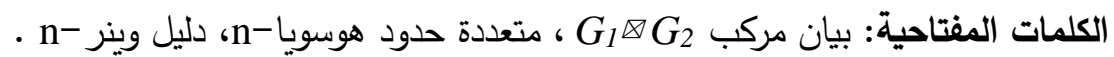

\title{
1. Introduction:
}

We follow the terminology of $[5,6,7,8]$. Let $\mathrm{v}$ be a vertex of a connected graph $\mathrm{G}$, and $\mathrm{S}$ be an $(\mathrm{n}-1)$ subset of $\mathrm{V}(\mathrm{G}), \mathrm{n} \geq 2$, then the $\mathrm{n}$-distance $\mathrm{d}_{\mathrm{n}}(\mathrm{v}, \mathrm{S})$ is defined [3] by

$\mathrm{d}_{\mathrm{n}}(\mathrm{v}, \mathrm{S})=\min \{\mathrm{d}(\mathrm{v}, \mathrm{u}): \mathrm{u} \in \mathrm{S}\}$.

The $\mathbf{n - d i a m e t e r}$ of $\mathbf{G}$ is defined by $\operatorname{diam}_{\mathrm{n}} \mathrm{G}=\max \left\{\mathrm{d}_{\mathrm{n}}(\mathrm{v}, \mathrm{S}): \mathrm{v} \in \mathrm{V}(\mathrm{G}),|\mathrm{S}|=\mathrm{n}-1, \mathrm{~S} \subseteq \mathrm{V}(\mathrm{G})\right\}$.

The $\mathbf{n}$-Wiener index of $\mathbf{G}$ is defined by 


$$
\mathrm{W}_{\mathrm{n}}(\mathrm{G})=\sum_{(\mathrm{v}, \mathrm{S})} \mathrm{d}_{\mathrm{n}}(\mathrm{v}, \mathrm{S}) \text {. }
$$

The $\mathbf{n - H o s o y a}$ polynomial of a connected graph $\mathbf{G}$ of order $\mathbf{p}$ is defined by

$$
\mathrm{H}_{\mathrm{n}}(\mathrm{G} ; \mathrm{x})=\sum_{\mathrm{k}=0}^{\delta_{\mathrm{n}}} \mathrm{C}_{\mathrm{n}}(\mathrm{G}, \mathrm{k}) \mathrm{x}^{\mathrm{k}} \text {, }
$$

where $2 \leq \mathrm{n} \leq \mathrm{p}, \delta_{\mathrm{n}}$ is the $\mathrm{n}$-diameter of $\mathrm{G}$, and $\mathrm{C}_{\mathrm{n}}(\mathrm{G}, \mathrm{k})$ is the number of order pairs $(\mathrm{v}, \mathrm{S}), \mathrm{v} \in \mathrm{V}(\mathrm{G}), \mathrm{S} \subseteq \mathrm{V}(\mathrm{G}),|\mathrm{S}|=\mathrm{n}-1$, such that $\mathrm{d}_{\mathrm{n}}(\mathrm{v}, \mathrm{S})=\mathrm{k}$.

One can easily show that

$$
\mathrm{C}_{\mathrm{n}}(\mathrm{G}, 0)=\mathrm{p}\left(\begin{array}{l}
\mathrm{p}-1 \\
\mathrm{n}-2
\end{array}\right), \mathrm{C}_{\mathrm{n}}(\mathrm{G}, 1)=\mathrm{p}\left(\begin{array}{c}
\mathrm{p}-1 \\
\mathrm{n}-1
\end{array}\right)-\sum_{\mathrm{v} \in \mathrm{V}(\mathrm{G})}\left(\begin{array}{c}
\mathrm{p}-1-\operatorname{deg} \mathrm{v} \\
\mathrm{n}-1
\end{array}\right) \text {. }
$$

The $\mathbf{n - H o s o y a}$ polynomial of a vertex $\mathbf{v}$ in $\mathbf{G}$, denoted by $H_{n}(v, G ; x)$, is defined [3] by

$$
\mathrm{H}_{\mathrm{n}}(\mathrm{v}, \mathrm{G} ; \mathrm{x})=\sum_{\mathrm{k} \geq 0} \mathrm{C}_{\mathrm{n}}(\mathrm{v}, \mathrm{G}, \mathrm{k}) \mathrm{x}^{\mathrm{k}} \text {, }
$$

where $C_{n}(v, G, k)$ is the number of $(n-1)$-subsets of vertices $S$ such that $d_{n}(v, S)=k$. It is clear that for each $\mathrm{k}, 0 \leq \mathrm{k} \leq \delta_{\mathrm{n}}$,

$$
\mathrm{C}_{\mathrm{n}}(\mathrm{G}, \mathrm{k})=\sum_{\mathrm{v} \in \mathrm{V}(\mathrm{G})} \mathrm{C}_{\mathrm{n}}(\mathrm{v}, \mathrm{G}, \mathrm{k})
$$

and

$$
\mathrm{H}_{\mathrm{n}}(\mathrm{G} ; \mathrm{x})=\sum_{\mathrm{v} \in \mathrm{V}(\mathrm{G})} \mathrm{H}_{\mathrm{n}}(\mathrm{v}, \mathrm{G} ; \mathrm{x}) \text {, }
$$

Let $\mathrm{T}$ be a non-empty subset of vertices of $\mathrm{G}$. We define

$$
\mathrm{C}_{\mathrm{n}}(\mathrm{T}, \mathrm{G}, \mathrm{k})=\sum_{\mathrm{v} \in \mathrm{T}} \mathrm{C}_{\mathrm{n}}(\mathrm{v}, \mathrm{G}, \mathrm{k}) \text {. }
$$

We shall use this notation in our proofs.

Finally, if $n=2$, then from $(1.5)$, we get

$\mathrm{C}_{2}(\mathrm{G}, 0)=\mathrm{p}=\mathrm{d}(\mathrm{G}, 0)$, and $\mathrm{C}_{2}(\mathrm{G}, 1)=\mathrm{p}(\mathrm{p}-1)-\sum_{\mathrm{v} \in \mathrm{V}(\mathrm{G})}(\mathrm{p}-1-\operatorname{deg} \mathrm{v})=\sum_{\mathrm{v} \in \mathrm{V}(\mathrm{G})} \operatorname{deg} \mathrm{v}=2 \mathrm{q}$,

then $\mathrm{d}(\mathrm{G}, 1)=\frac{1}{2} \mathrm{C}_{2}(\mathrm{G}, 1)=\mathrm{q}$. Also, we notice that $\mathrm{d}(\mathrm{G}, \mathrm{k})=\frac{1}{2} \mathrm{C}_{2}(\mathrm{G}, \mathrm{k}), \mathrm{k} \geq 2$.

Hence $\mathrm{H}(\mathrm{G} ; \mathrm{x})=\frac{1}{2} \mathrm{H}_{2}(\mathrm{G} ; \mathrm{x})$.

In [2], H. G. Ahmed gave the following result :

Lemma: Let $v$ be any vertex of a connected graph $G$. If there are $r$ vertices of distance $\mathrm{k} \geq 1$ from $\mathrm{v}$, and there are $\mathrm{s}$ vertices of distance more than $\mathrm{k}$ from $\mathrm{v}$, then $\mathrm{C}_{\mathrm{n}}(\mathrm{v}, \mathrm{G}, \mathrm{k})=\left(\begin{array}{c}\mathrm{r}+\mathrm{s} \\ \mathrm{n}-1\end{array}\right)-\left(\begin{array}{c}\mathrm{s} \\ \mathrm{n}-1\end{array}\right)$.

In 2007, H.O. Abdulla [1] and A.S. Aziz [4] defined the composite graph $\mathrm{G}_{1} \bowtie \mathrm{G}_{2}$ as follows:

Let $\mathrm{G}_{1}$ and $\mathrm{G}_{2}$ be disjoint connected graphs, and let $\mathrm{u}_{1} \mathrm{u}_{2} \in \mathrm{E}\left(\mathrm{G}_{1}\right)$ and $\mathrm{v}_{1} \mathrm{v}_{2} \in \mathrm{E}\left(\mathrm{G}_{2}\right)$, then the composite graph $\mathrm{G}_{1} \otimes \mathrm{G}_{2}$ is the graph constructed 
from $\mathrm{G}_{1}$ and $\mathrm{G}_{2}$ by adding the edges $\mathrm{u}_{1} \mathrm{v}_{1}, \mathrm{u}_{1} \mathrm{v}_{2}, \mathrm{u}_{2} \mathrm{v}_{1}$, and $\mathrm{u}_{2} \mathrm{v}_{2}$. It is clear that $\mathrm{p}\left(\mathrm{G}_{1} \otimes \mathrm{G}_{2}\right)=\mathrm{p}\left(\mathrm{G}_{1}\right)+\mathrm{p}\left(\mathrm{G}_{2}\right)$ and $\mathrm{q}\left(\mathrm{G}_{1} \otimes \mathrm{G}_{2}\right)=\mathrm{q}\left(\mathrm{G}_{1}\right)+\mathrm{q}\left(\mathrm{G}_{2}\right)+4$.

In this paper, we obtain n-Hosoya polynomials, n-Wiener indices, Hosoya polynomials and Wiener indices of the composite of some special graphs .

\section{The Composite Graph $K_{\alpha} \otimes K_{\beta}$ :}

Let $\mathrm{K}_{\alpha}$ and $\mathrm{K}_{\beta}$ be complete graphs of orders $\alpha, \alpha \geq 2$ and $\beta, \beta \geq 2$ respectively .The composite graph $\mathrm{K}_{\alpha} \otimes \mathrm{K}_{\beta}$ is depicted in Fig. 2.1. We assume, without loss of generality that $\alpha \geq \beta$.

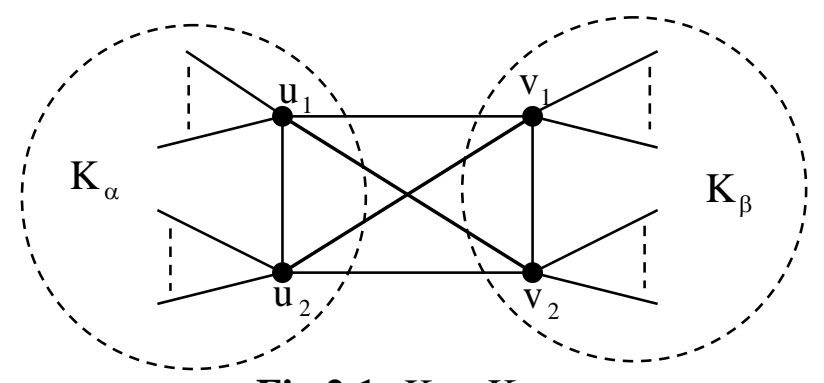

Fig.2.1. $\mathrm{K}_{\alpha} \otimes \mathrm{K}_{\beta}$

We notice that the $\mathrm{n}$-diameter of $\mathrm{K}_{\alpha} \otimes \mathrm{K}_{\beta}$ is

$$
\operatorname{diam}_{\mathrm{n}} \mathrm{K}_{\alpha} \otimes \mathrm{K}_{\beta}=\left[\begin{array}{ll}
3, & \text { if } 2 \leq \mathrm{n} \leq \alpha-1 \\
2 \text { or } 1, & \text { if } \mathrm{n} \geq \alpha
\end{array}\right.
$$

To simplify the notation, we denote $\mathrm{K}_{\alpha} \otimes \mathrm{K}_{\beta}$ by $\mathrm{G}$.From ,Fig.2.1, we find that

$C_{n}(G, 3)=(\alpha-2)\left(\begin{array}{l}\beta-2 \\ n-1\end{array}\right)+(\beta-2)\left(\begin{array}{l}\alpha-2 \\ n-1\end{array}\right)$.

Since, $\operatorname{diam}_{n} K_{\alpha} \otimes K_{\beta} \leq 3$, then from (1.5)

$\mathrm{C}_{\mathrm{n}}(\mathrm{G}, 2)+\mathrm{C}_{\mathrm{n}}(\mathrm{G}, 3)=\sum_{\mathrm{v} \in \mathrm{V}(\mathrm{G})}\left(\begin{array}{c}\mathrm{p}-1-\mathrm{deg} \mathrm{v} \\ \mathrm{n}-1\end{array}\right)$.

Hence,

$C_{n}(G, 2)=(\alpha-2)\left(\begin{array}{c}\beta \\ n-1\end{array}\right)+(\beta-2)\left(\begin{array}{c}\alpha \\ n-1\end{array}\right)-(\alpha-4)\left(\begin{array}{l}\beta-2 \\ n-1\end{array}\right)-(\beta-4)\left(\begin{array}{l}\alpha-2 \\ n-1\end{array}\right)$.

From (1.5) we get

$$
\mathrm{C}_{\mathrm{n}}(\mathrm{G}, 1)=\mathrm{p}\left(\begin{array}{l}
\mathrm{p}-1 \\
\mathrm{n}-1
\end{array}\right)-(\alpha-2)\left(\begin{array}{c}
\beta \\
\mathrm{n}-1
\end{array}\right)-(\beta-2)\left(\begin{array}{c}
\alpha \\
\mathrm{n}-1
\end{array}\right)-2\left(\begin{array}{l}
\beta-2 \\
\mathrm{n}-1
\end{array}\right)-2\left(\begin{array}{l}
\alpha-2 \\
\mathrm{n}-1
\end{array}\right) .
$$

From (2.1), (2.2), and (2.3) we have the next proposition:

Proposition 2.1: For $2 \leq \mathrm{n} \leq \mathrm{p}=\alpha+\beta, \alpha, \beta \geq 2$, then

$H_{n}(G ; x)=p\left(\begin{array}{l}p-1 \\ n-2\end{array}\right)+\sum_{k=1}^{3} C_{n}(G, k) x^{k}$,

where, $\mathrm{C}_{\mathrm{n}}(\mathrm{G}, \mathrm{k}), 1 \leq \mathrm{k} \leq 3$, are given in (2.1), (2.2), and (2.3).

And $W_{n}(G)=p\left(\begin{array}{l}p-1 \\ n-1\end{array}\right)+\alpha\left(\begin{array}{l}\beta-2 \\ n-1\end{array}\right)+\beta\left(\begin{array}{l}\alpha-2 \\ n-1\end{array}\right)+(\alpha-2)\left(\begin{array}{c}\beta \\ n-1\end{array}\right)+(\beta-2)\left(\begin{array}{c}\alpha \\ n-1\end{array}\right)$. 
From Proposition 2.1 and (1.10), we get the next corollary.

Corollary 2.2: The Hosoya polynomial of the graph $G$ of order $\alpha+\beta, \alpha, \beta \geq 2$, is given by :

$\mathrm{H}(\mathrm{G} ; \mathrm{x})=(\alpha+\beta)+\frac{1}{2}[\alpha(\alpha-1)+\beta(\beta-1)+8] \mathrm{x}+2[\alpha+\beta-4] \mathrm{x}^{2}+(\alpha-2)(\beta-2) \mathrm{x}^{3}$.

And Wiener index of $\mathrm{G}$ is

$\mathrm{W}(\mathrm{G})=\frac{1}{2}[\alpha(\alpha-5)+\beta(\beta-5)+6 \alpha \beta]$.

\section{The Composite Graph $\mathrm{K}_{\alpha} \otimes \mathrm{K}_{\beta, \gamma}$ :}

Let $\mathrm{K}_{\alpha}$ be a complete graph of order $\alpha, \alpha \geq 2$ and $\mathrm{K}_{\beta, \gamma}$ be a complete bipartite graph of order $\beta+\gamma, \beta, \gamma \geq 2$, then $\mathrm{K}_{\alpha} \otimes \mathrm{K}_{\beta, \gamma}$ is depicted in Fig. 3.1.

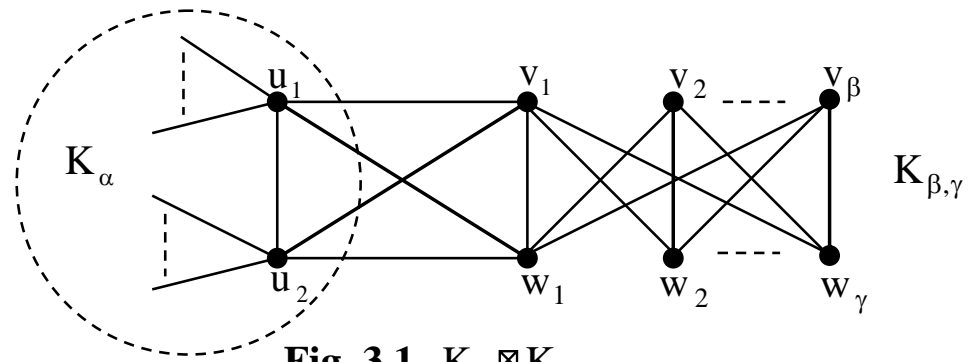

Fig. 3.1. $K_{\alpha} \otimes K_{\beta, \gamma}$

The order of $\mathrm{K}_{\alpha} \otimes \mathrm{K}_{\beta, \gamma}$ is $\mathrm{p}=\alpha+\beta+\gamma$, the size is $\mathrm{q}=\frac{1}{2}\{\alpha(\alpha-1)+2 \gamma \beta+8\}$, and the diameter is 3 for $\alpha \geq 3$ and $\beta, \gamma \geq 2$. We denote $K_{\alpha} \otimes K_{\beta, \gamma}$ by $G^{\prime}$.

The $n$-diameter of $\mathrm{G}^{\prime}$ is given by $\operatorname{diam}_{n} \mathrm{G}^{\prime}=\left[\begin{array}{ll}3, & \text { if } 2 \leq \mathrm{n} \leq \max \{\alpha-1, \beta, \gamma\} \\ 2 \text { or } 1, & \text { if } \mathrm{n}>\max \{\alpha-1, \beta, \gamma\}\end{array}\right.$.

In the next proposition, we obtain the n-Hosoya polynomial of $\mathrm{G}^{\prime}$ :

Proposition 3.1: For $2 \leq \mathrm{n} \leq \mathrm{p}=\alpha+\beta+\gamma, \alpha, \beta, \gamma \geq 2$, then

$$
\mathrm{H}_{\mathrm{n}}\left(\mathrm{G}^{\prime} ; \mathrm{x}\right)=\mathrm{p}\left(\begin{array}{l}
\mathrm{p}-1 \\
\mathrm{n}-2
\end{array}\right)+\sum_{\mathrm{k}=1}^{3} \mathrm{C}_{\mathrm{n}}\left(\mathrm{G}^{\prime}, \mathrm{k}\right) \mathrm{x}^{\mathrm{k}} \text {, }
$$

where,

$$
\begin{gathered}
\mathrm{C}_{\mathrm{n}}\left(\mathrm{G}^{\prime}, 1\right)=\mathrm{p}\left(\begin{array}{c}
\mathrm{p}-1 \\
\mathrm{n}-1
\end{array}\right)-(\alpha-2)\left(\begin{array}{c}
\beta+\gamma \\
\mathrm{n}-1
\end{array}\right)-(\beta-1)\left(\begin{array}{c}
\alpha+\beta-1 \\
\mathrm{n}-1
\end{array}\right)-(\gamma-1)\left(\begin{array}{c}
\alpha+\gamma-1 \\
\mathrm{n}-1
\end{array}\right) \\
-2\left(\begin{array}{c}
\beta+\gamma-2 \\
\mathrm{n}-1
\end{array}\right)-\left(\begin{array}{c}
\alpha+\beta-3 \\
\mathrm{n}-1
\end{array}\right)-\left(\begin{array}{c}
\alpha+\gamma-3 \\
\mathrm{n}-1
\end{array}\right) . \\
\mathrm{C}_{\mathrm{n}}\left(\mathrm{G}^{\prime}, 2\right)=(\alpha-2)\left(\begin{array}{c}
\beta+\gamma \\
\mathrm{n}-1
\end{array}\right)+(\beta-1)\left(\begin{array}{c}
\alpha+\beta-1 \\
\mathrm{n}-1
\end{array}\right)+(\gamma-1)\left(\begin{array}{c}
\alpha+\gamma-1 \\
\mathrm{n}-1
\end{array}\right)+\left(\begin{array}{c}
\alpha+\beta-3 \\
\mathrm{n}-1
\end{array}\right) \\
+\left(\begin{array}{c}
\alpha+\gamma-3 \\
\mathrm{n}-1
\end{array}\right)-(\alpha-4)\left(\begin{array}{c}
\beta+\gamma-2 \\
\mathrm{n}-1
\end{array}\right)-(\beta+\gamma-2)\left(\begin{array}{c}
\alpha-2 \\
\mathrm{n}-1
\end{array}\right) .
\end{gathered}
$$


$C_{n}\left(G^{\prime}, 3\right)=(\alpha-2)\left(\begin{array}{c}\beta+\gamma-2 \\ n-1\end{array}\right)+(\beta+\gamma-2)\left(\begin{array}{l}\alpha-2 \\ n-1\end{array}\right)$.

Proof: From (1.5), we get (3.1.1), and from Fig. 3.1, we have

$C_{n}\left(G^{\prime}, 3\right)=(\alpha-2)\left(\begin{array}{c}\beta+\gamma-2 \\ n-1\end{array}\right)+(\beta+\gamma-2)\left(\begin{array}{l}\alpha-2 \\ n-1\end{array}\right)$.

Since $\operatorname{diam}_{\mathrm{n}} \mathrm{G}^{\prime} \leq 3$, then,

$$
\begin{array}{r}
C_{n}\left(G^{\prime}, 2\right)+C_{n}\left(G^{\prime}, 3\right)=\sum_{v \in V(G)}\left(\begin{array}{c}
p-1-\operatorname{deg} v \\
n-1
\end{array}\right)=(\alpha-2)\left(\begin{array}{c}
\beta+\gamma \\
n-1
\end{array}\right)+(\beta-1)\left(\begin{array}{c}
\alpha+\beta-1 \\
n-1
\end{array}\right) \\
+(\gamma-1)\left(\begin{array}{c}
\alpha+\gamma-1 \\
n-1
\end{array}\right)+2\left(\begin{array}{c}
\beta+\gamma-2 \\
n-1
\end{array}\right)+\left(\begin{array}{c}
\alpha+\beta-3 \\
n-1
\end{array}\right)+\left(\begin{array}{c}
\alpha+\gamma-3 \\
n-1
\end{array}\right)
\end{array}
$$

This completes the proof.

Corollary 3.2: : For $2 \leq \mathrm{n} \leq \mathrm{p}=\alpha+\beta+\gamma, \alpha \geq 2$ and $\beta, \gamma \geq 2$, we have

$$
\begin{array}{r}
\mathrm{W}_{\mathrm{n}}\left(\mathrm{G}^{\prime}\right)=\mathrm{p}\left(\begin{array}{c}
\mathrm{p}-1 \\
\mathrm{n}-1
\end{array}\right)+(\alpha-2)\left(\begin{array}{c}
\beta+\gamma \\
\mathrm{n}-1
\end{array}\right)+(\beta-1)\left(\begin{array}{c}
\alpha+\beta-1 \\
\mathrm{n}-1
\end{array}\right)+(\gamma-1)\left(\begin{array}{c}
\alpha+\gamma-1 \\
\mathrm{n}-1
\end{array}\right) \\
+\left(\begin{array}{c}
\alpha+\beta-3 \\
\mathrm{n}-1
\end{array}\right)+\left(\begin{array}{c}
\alpha+\gamma-3 \\
\mathrm{n}-1
\end{array}\right)+\alpha\left(\begin{array}{c}
\beta+\gamma-2 \\
\mathrm{n}-1
\end{array}\right)+(\beta+\gamma-2)\left(\begin{array}{l}
\alpha-2 \\
\mathrm{n}-1
\end{array}\right) .
\end{array}
$$

From Proposition 3.1 and (1.10), we get the next corollary.

Corollary 3.3: For $\alpha, \beta, \gamma \geq 2$, we have :

$$
\begin{aligned}
\mathrm{H}\left(\mathrm{G}^{\prime} ; \mathrm{x}\right)=(\alpha+\beta & \beta+\gamma)+\frac{1}{2}[\alpha(\alpha-1)+2 \beta \gamma+8] \mathrm{x}+\frac{1}{2}[4(\alpha-4)+\beta(\beta+3)+\gamma(\gamma+3)] \mathrm{x}^{2} \\
& +(\alpha-2)(\beta+\gamma-2) \mathrm{x}^{3}
\end{aligned}
$$

And, Wiener index of $\mathrm{G}^{\prime}$ is $\mathrm{W}\left(\mathrm{G}^{\prime}\right)=\frac{1}{2} \alpha(\alpha-5)+\beta(\beta-3)+\gamma(\gamma-3)+3 \alpha(\beta+\gamma)+\beta \gamma$

\section{The Composite Graph $\mathrm{K}_{\alpha} \otimes \mathrm{W}_{\beta}$ :}

Let $\mathrm{K}_{\alpha}, \alpha \geq 2$, and $\mathrm{W}_{\beta}, \beta \geq 4$ be complete and wheel graphs respectively, then the composite graph $\mathrm{K}_{\alpha} \otimes \mathrm{W}_{\beta}$ has order $\mathrm{p}=\alpha+\beta, \mathrm{q}=\frac{1}{2}\{\alpha(\alpha-1)+4(\beta+1)\}$, and diameter 4 , for $\beta \geq 6$ and $\alpha \geq 3$.

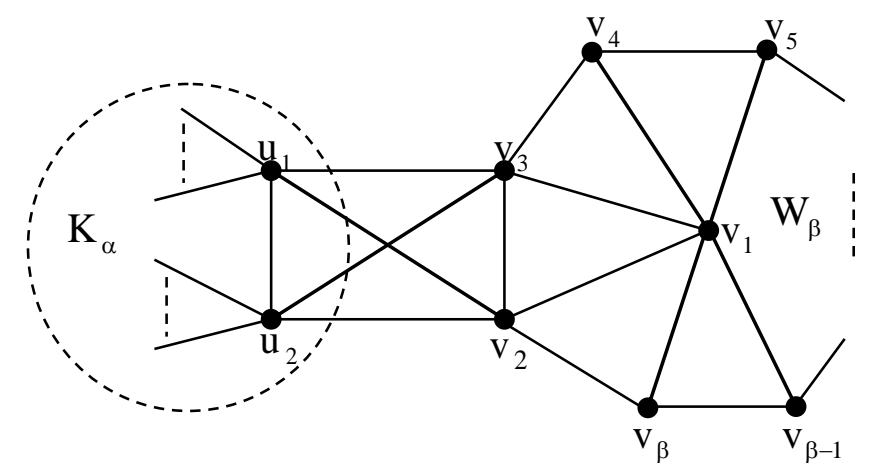

Fig.4.1 $\mathrm{K}_{\alpha} \otimes \mathrm{W}_{\beta}$ 
We denote $\mathrm{K}_{\alpha} \otimes \mathrm{W}_{\beta}$ by $\mathrm{G}^{\prime \prime}$. From Fig.4.1, with $\alpha \geq 3, \beta \geq 6$ we notice that :

$\operatorname{diam}_{n} G^{\prime \prime}=\left[\begin{array}{ll}4, & \text { if } 2 \leq \mathrm{n} \leq \max \{\alpha-1, \beta-4\}, \\ 3, & \text { if } \max \{\alpha, \beta-3\} \leq \mathrm{n} \leq \max \{\alpha+1, \beta-1\}, . \\ 2 \text { or } 1, & \text { if } \mathrm{n}>\max \{\alpha+1, \beta-1\} .\end{array}\right.$

In the next proposition, we obtain the n-Hosoya polynomial of $\mathrm{G}^{\prime \prime}$ :

Proposition 4.1: For $2 \leq \mathrm{n} \leq \mathrm{p}=\alpha+\beta+\gamma, \alpha \geq 3, \beta \geq 6$, we have

$\mathrm{H}_{\mathrm{n}}\left(\mathrm{G}^{\prime \prime} ; \mathrm{x}\right)=\mathrm{p}\left(\begin{array}{c}\mathrm{p}-1 \\ \mathrm{n}-2\end{array}\right)+\sum_{\mathrm{k}=1}^{4} \mathrm{C}_{\mathrm{n}}\left(\mathrm{G}^{\prime \prime}, \mathrm{k}\right) \mathrm{x}^{\mathrm{k}}$,

where,

$$
\begin{aligned}
& C_{n}\left(G^{\prime \prime}, 1\right)=p\left(\begin{array}{l}
p-1 \\
n-1
\end{array}\right)-(\beta-3)\left(\begin{array}{l}
p-4 \\
n-1
\end{array}\right)-2\left(\begin{array}{l}
p-6 \\
n-1
\end{array}\right)-\left(\begin{array}{c}
\alpha \\
n-1
\end{array}\right)-2\left(\begin{array}{l}
\beta-2 \\
n-1
\end{array}\right)-(\alpha-2)\left(\begin{array}{c}
\beta \\
n-1
\end{array}\right), \\
& C_{n}\left(G^{\prime \prime}, 2\right)=(\beta-3)\left(\begin{array}{l}
p-4 \\
n-1
\end{array}\right)+2\left(\begin{array}{l}
p-6 \\
n-1
\end{array}\right)+(\alpha-2)\left(\begin{array}{c}
\beta \\
n-1
\end{array}\right)-(\beta-6)\left(\begin{array}{c}
\alpha \\
n-1
\end{array}\right)-(\alpha-4)\left(\begin{array}{l}
\beta-2 \\
n-1
\end{array}\right) \\
& C_{n}\left(G^{\prime \prime}, 3\right)=(\alpha-2)\left(\begin{array}{l}
\beta-5 \\
n-1
\end{array}\right)-3\left(\begin{array}{l}
\alpha-2 \\
n-1
\end{array}\right), \\
& C_{n}\left(G^{\prime \prime}, 4\right)=(\alpha-2)\left(\begin{array}{l}
\beta-5 \\
n-1
\end{array}\right)+(\beta-5)\left(\begin{array}{l}
\alpha-2 \\
n-1
\end{array}\right) .
\end{aligned}
$$

Proof: From (1.5), we get $C_{n}\left(G^{\prime \prime}, 1\right)$. To find the coefficient $C_{n}\left(G^{\prime \prime}, 2\right)$, we , first find $\mathrm{C}_{\mathrm{n}}\left(\mathrm{G}^{\prime \prime}, \mathrm{k}\right), \mathrm{k}=3,4$.

For $\mathrm{k}=3$, there are three vertices namely, $\mathrm{v}_{1}, \mathrm{v}_{4}, \mathrm{v}_{\beta}$ of distance 3 from $\mathrm{u}_{\mathrm{i}}$, $3 \leq \mathrm{i} \leq \alpha$, and there are $\beta-5$ vertices of distance more than 3 from $\mathrm{u}_{\mathrm{i}}$. Hence, by

$C_{n}\left(u_{i}, G^{\prime \prime}, 3\right)=\left(\begin{array}{l}\beta-2 \\ n-1\end{array}\right)-\left(\begin{array}{l}\beta-5 \\ n-1\end{array}\right), 3 \leq i \leq \alpha$.

But, there are two vertices namely $\mathrm{u}_{1}$ and $\mathrm{u}_{2}$ of distance 3 from $\mathrm{v}_{\mathrm{i}}, 5 \leq \mathrm{i} \leq \beta-1$, and there are $\alpha-2$ vertices of distance more than 3 from $v_{i}$. Hence, by (1.11)

$\mathrm{C}_{\mathrm{n}}\left(\mathrm{v}_{\mathrm{i}}, \mathrm{G}^{\prime \prime}, 3\right)=\left(\begin{array}{c}\alpha \\ \mathrm{n}-1\end{array}\right)-\left(\begin{array}{c}\alpha-2 \\ \mathrm{n}-1\end{array}\right), 5 \leq \mathrm{i} \leq \beta-1$.

Finally, there are $\beta-5$ vertices namely $, v_{5}, v_{6}, \ldots, v_{\beta-1}$ of distance 3 from $u_{i}$, $\mathrm{i}=1,2$, and there is no vertex of distance more than 3 from $u_{i}$, and there are $\alpha-2$ vertices, namely $\mathrm{u}_{3}, \mathrm{u}_{4}, \ldots, \mathrm{u}_{\alpha}$ of distance 3 from $\mathrm{v}_{\mathrm{i}}, \mathrm{i}=1,4, \beta$, and there is no vertex of distance more than 3 from $v_{i}$. Hence,

$C_{n}\left(u_{i}, G^{\prime \prime}, 3\right)=\left(\begin{array}{l}\beta-5 \\ n-1\end{array}\right), i=1,2$,

and, 
$\mathrm{C}_{\mathrm{n}}\left(\mathrm{v}_{\mathrm{i}}, \mathrm{G}^{\prime \prime}, 3\right)=\left(\begin{array}{c}\alpha-2 \\ \mathrm{n}-1\end{array}\right), \mathrm{i}=1,4, \beta$.

Hence, from (4.1.1)-(4.1.4) we get $\mathrm{C}_{\mathrm{n}}\left(\mathrm{G}^{\prime \prime}, 3\right)$.

Now , for $\mathrm{k}=4$, there are $\beta-5$ vertices , namely $\mathrm{v}_{5}, \mathrm{v}_{6}, \ldots, \mathrm{v}_{\beta-1}$ of distance 4 from $u_{i}, 3 \leq i \leq \alpha$, and there is no vertex of distance more than 4 from $u_{i}$, then

$\mathrm{C}_{\mathrm{n}}\left(\mathrm{u}_{\mathrm{i}}, \mathrm{G}^{\prime \prime}, 4\right)=\left(\begin{array}{c}\beta-5 \\ \mathrm{n}-1\end{array}\right), 3 \leq \mathrm{i} \leq \alpha$.

And, there are $\alpha-2$ vertices, namely $\mathrm{u}_{3}, \mathrm{u}_{4}, \ldots, \mathrm{u}_{\alpha}$ of distance 4 from $\mathrm{v}_{\mathrm{i}}$, $5 \leq \mathrm{i} \leq \beta-1$, and there is no vertex of distance more than 4 from $v_{i}$, then

$\mathrm{C}_{\mathrm{n}}\left(\mathrm{v}_{\mathrm{i}}, \mathrm{G}^{\prime \prime}, 4\right)=\left(\begin{array}{c}\alpha-2 \\ \mathrm{n}-1\end{array}\right), 5 \leq \mathrm{i} \leq \beta-1$.

Hence, from (4.1.5) and (4.1.6) we get $C_{n}\left(G^{\prime \prime}, 4\right)$.

From the relation $\sum_{\mathrm{k}=2}^{4} \mathrm{C}\left(\mathrm{G}^{\prime \prime}, \mathrm{K}\right)=\sum_{\mathrm{v} \in \mathrm{V}\left(\mathrm{G}^{\prime \prime}\right)}\left(\begin{array}{c}\mathrm{p}-1-\operatorname{deg} \mathrm{v} \\ \mathrm{n}-1\end{array}\right)$, we obtain $\mathrm{C}_{\mathrm{n}}\left(\mathrm{G}^{\prime \prime}, 2\right)$ as it is given in Proposition 4.1.

\section{Remark 1:}

- If $\alpha=2$ and $\beta \geq 6$, Proposition 4.1 holds with $\mathrm{C}_{\mathrm{n}}\left(\mathrm{G}^{\prime \prime}, 4\right)=0$.

- If $\alpha \geq 2$ and $\beta=5$, Proposition 4.1 holds with $\mathrm{C}_{\mathrm{n}}\left(\mathrm{G}^{\prime \prime}, 4\right)=0$.

- If $\alpha \geq 2$ and $\beta=4$, we have $K_{\alpha} \otimes K_{4}$ which is given in Proposition 2.1.

Corollary 4.2: For $2 \leq \mathrm{n} \leq \mathrm{p}=\alpha+\beta+\gamma, \alpha \geq 3, \beta \geq 6$, we have

$$
\begin{array}{r}
\mathrm{W}_{\mathrm{n}}\left(\mathrm{G}^{\prime \prime}\right)=\mathrm{p}\left(\begin{array}{l}
\mathrm{p}-1 \\
\mathrm{n}-1
\end{array}\right)+(\beta-3)\left(\begin{array}{l}
\mathrm{p}-4 \\
\mathrm{n}-1
\end{array}\right)+2\left(\begin{array}{l}
\mathrm{p}-6 \\
\mathrm{n}-1
\end{array}\right)+(\alpha-2)\left(\begin{array}{c}
\beta \\
\mathrm{n}-1
\end{array}\right)+(\beta-4)\left(\begin{array}{c}
\alpha \\
\mathrm{n}-1
\end{array}\right) \\
+\alpha\left(\begin{array}{l}
\beta-2 \\
\mathrm{n}-1
\end{array}\right)+(\beta-2)\left(\begin{array}{l}
\alpha-2 \\
\mathrm{n}-1
\end{array}\right)+\alpha\left(\begin{array}{l}
\beta-5 \\
\mathrm{n}-1
\end{array}\right) .
\end{array}
$$

From Proposition 4.1 and (1.10), we get the next corollary.

Corollary 4.3: For, $\alpha \geq 2, \beta \geq 5$, the Hosoya polynomial of $\mathrm{G}^{\prime \prime}$ of order $\alpha+\beta$ is given by:

$$
\begin{array}{r}
\mathrm{H}\left(\mathrm{G}^{\prime \prime} ; \mathrm{x}\right)=(\alpha+\beta)+\frac{1}{2}[\alpha(\alpha-1)+4(\beta+1)] \mathrm{x}+\frac{1}{2}[4(\alpha+2)+\beta(\beta-5)] \mathrm{x}^{2}+[3 \alpha+2 \beta-16] \mathrm{x}^{3} \\
+(\alpha-2)(\beta-5) \mathrm{x}^{4}
\end{array}
$$

And, Wiener index of $\mathrm{G}^{\prime \prime}$ is

$\mathrm{W}\left(\mathrm{G}^{\prime \prime}\right)=\frac{1}{2} \alpha(\alpha-15)+\beta(\beta-5)+4 \alpha \beta+2$.

Remark 2 : If $\alpha \geq 2$ and $\beta=4$, we have

$\mathrm{H}\left(\mathrm{G}^{\prime \prime} ; \mathrm{x}\right)=(\alpha+4)+\frac{1}{2}[\alpha(\alpha-1)+20] \mathrm{x}+2 \alpha \mathrm{x}^{2}+2(\alpha-2) \mathrm{x}^{3}$,

and, 
$\mathrm{W}\left(\mathrm{G}^{\prime \prime}\right)=\frac{1}{2} \alpha(\alpha+19)-2$.

\section{The Composite Graph $\mathrm{K}_{\alpha} \otimes \mathrm{C}_{\beta}$ :}

Let $\mathrm{C}_{\beta}$ be a cycle graph of order $\beta, \beta \geq 4$ and let $\mathrm{v}_{1} \mathrm{v}_{\beta} \in \mathrm{E}\left(\mathrm{C}_{\beta}\right)$ and, $\mathrm{K}_{\alpha}$ be a complete graph of order $\alpha, \alpha \geq 2$ and let $\mathrm{u}_{1} \mathrm{u}_{2} \in \mathrm{E}\left(\mathrm{K}_{\alpha}\right)$, then the composite graph $\mathrm{K}_{\alpha} \otimes \mathrm{C}_{\beta}$ has order $\mathrm{p}=\alpha+\beta$, size $\mathrm{q}=\frac{1}{2}\{\alpha(\alpha-1)+2 \beta+8\}$, and diameter $\left\lceil\frac{\beta}{2}\right\rceil+1$, as depicted in Fig.5.1.

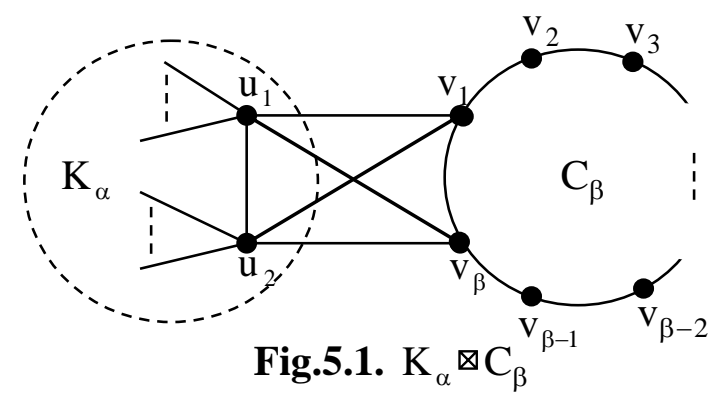

We denote $\mathrm{K}_{\alpha} \otimes \mathrm{C}_{\beta}$ by $\mathrm{G}^{\prime \prime \prime}$. The n-diameter of $\mathrm{G}^{\prime \prime \prime}$ is determined in the following proposition:

Proposition 5.1: For $2 \leq \mathrm{n} \leq \mathrm{p}=\alpha+\beta+\gamma, \alpha \geq 3, \beta \geq 4$, then

$$
\operatorname{diam}_{\mathrm{n}} \mathrm{G}^{\prime \prime \prime}=\left[\begin{array}{ll}
\left\lceil\frac{\beta}{2}\right\rceil+1, & \text { if } 2 \leq \mathrm{n} \leq \alpha-1, \\
\left\lceil\frac{\beta}{2}\right\rceil, & \text { if } \mathrm{n}=\alpha \text { or } \alpha+1, . \\
\left\lfloor\frac{\mathrm{p}-\mathrm{n}}{2}\right\rfloor+1, & \text { if } \alpha+2 \leq \mathrm{n} \leq \mathrm{p} .
\end{array}\right.
$$

Proof : Let $S$ be an (n-1) - subset of $V\left(G^{\prime \prime \prime}\right)$, and let w be a vertex of $V\left(G^{\prime \prime \prime}\right)$, such that $d_{n}(w, S)=\operatorname{diam}_{n} G^{\prime \prime \prime}$. Since the n-diameter is the maximum of the n-distances $d_{n}(v, S)$, $\mathrm{v} \in \mathrm{V}\left(\mathrm{G}^{\prime \prime \prime}\right), \mathrm{S} \subseteq \mathrm{V}\left(\mathrm{G}^{\prime \prime \prime}\right),|\mathrm{S}|=\mathrm{n}-1$, then $\mathrm{w}$ must be a vertex of $\mathrm{C}_{\beta}$ that is furthest from $\left\{\mathrm{v}_{1}, \mathrm{v}_{\beta}\right\}$, that is

$\mathrm{W}= \begin{cases}\mathrm{v}_{\frac{\beta}{2}} \text { or } \mathrm{v}_{\frac{\beta}{2}+1}, & \text { if } \beta \text { is even, } \\ \mathrm{v}_{\frac{\beta+1}{2}}, & \text { if } \beta \text { is odd, }\end{cases}$

and $S$ consists of the first $n-1$ vertices from the sequence $\mathrm{u}_{3}, \mathrm{u}_{4}, \ldots, \mathrm{u}_{\alpha}, \mathrm{u}_{1}, \mathrm{u}_{2}, \mathrm{v}_{1}, \mathrm{v}_{\beta}, \mathrm{v}_{2}, \mathrm{v}_{\beta-1}, \ldots$. Therefore :

$\mathrm{d}_{\mathrm{n}}(\mathrm{w}, \mathrm{S})=\left\{\begin{array}{l}{\left[\frac{\beta}{2}\right\rceil+1, \text { if } 2 \leq \mathrm{n} \leq \alpha-1, \mathrm{~S} \subseteq \mathrm{V}\left(\mathrm{K}_{\alpha}\right)-\left\{\mathrm{u}_{1}, \mathrm{u}_{2}\right\},} \\ {\left[\frac{\beta}{2}\right], \quad \text { if } \mathrm{n}=\alpha \text { or } \alpha+1, \mathrm{~S} \subseteq \mathrm{V}\left(\mathrm{K}_{\alpha}\right) .}\end{array}\right.$ 
Since $\operatorname{diam}_{n} C_{\beta}=\left\lfloor\frac{\beta-n}{2}\right\rfloor+1$, for $2 \leq \mathrm{n} \leq \beta,[1]$, then

$d_{n}(w, S)=\left\lfloor\frac{\beta-(n-\alpha)}{2}\right\rfloor+1$, if $\alpha+2 \leq n \leq \alpha+\beta$, for which

$\mathrm{S}=\mathrm{V}\left(\mathrm{K}_{\alpha}\right) \cup\left\{\mathrm{V}\left(\mathrm{C}_{\beta}\right)-\{\mathrm{w}\}\right\}$.

From (5.1.2) and (5.1.3), we get (5.1.1).

To find the coefficients of the n-Hosoya polynomial of the composite graph $\mathrm{G}^{\prime \prime \prime}$, we denote $\mathrm{V}\left(\mathrm{K}_{\alpha}\right)$ by $\mathrm{U}$, and $\mathrm{V}\left(\mathrm{C}_{\beta}\right)$ by $\mathrm{V}$, and notice, for $2 \leq \mathrm{k} \leq \operatorname{diam}_{\mathrm{n}} \mathrm{G}^{\prime \prime \prime}$, that $\mathrm{C}_{\mathrm{n}}\left(\mathrm{G}^{\prime \prime \prime}, \mathrm{k}\right)=\mathrm{C}_{\mathrm{n}}\left(\mathrm{U}, \mathrm{G}^{\prime \prime \prime}, \mathrm{k}\right)+\mathrm{C}_{\mathrm{n}}\left(\mathrm{V}, \mathrm{G}^{\prime \prime \prime}, \mathrm{k}\right)$.

In the next lemmas, we obtain the coefficients of the n-Hosoya polynomial of $\mathrm{G}^{\prime \prime \prime}$.

Lemma 5.2: For $3 \leq \mathrm{n} \leq \beta+1, \alpha \geq 3, \beta \geq 4$, and $2 \leq \mathrm{k} \leq\left\lfloor\frac{\beta-\mathrm{n}+1}{2}\right\rfloor+2$, we have

$\mathrm{C}_{\mathrm{n}}\left(\mathrm{U}, \mathrm{G}^{\prime \prime \prime}, \mathrm{k}\right)=\left\{\begin{array}{c}(\alpha-2)\left(\begin{array}{c}\beta-2 \mathrm{k}+4 \\ \mathrm{n}-1\end{array}\right)-(\alpha-4)\left(\begin{array}{c}\beta-2 \mathrm{k}+2 \\ \mathrm{n}-1\end{array}\right)-2\left(\begin{array}{c}\beta-2 \mathrm{k} \\ \mathrm{n}-1\end{array}\right), \text { if } 2 \leq \mathrm{k} \leq\left\lfloor\frac{\beta-\mathrm{n}+1}{2}\right\rfloor+1, \mathrm{n}<\beta \\ (\alpha-2) \mathrm{r}, \text { if } \mathrm{k}=\left\lfloor\frac{\beta-\mathrm{n}+1}{2}\right\rfloor+2,\end{array}\right.$.

where $r=\left[\begin{array}{l}1, \text { if } \beta-n+1 \text { is even , } \\ n, \text { if } \beta-n+1 \text { is odd . }\end{array}\right.$

Proof: For $3 \leq \mathrm{n} \leq \beta+1$ and $2 \leq \mathrm{k} \leq\left\lfloor\frac{\beta-\mathrm{n}+1}{2}\right\rfloor+2$, there are two vertices, namely $\mathrm{v}_{\mathrm{k}-1}$ and $\mathrm{v}_{\beta-\mathrm{k}+2}$ of distance $\mathrm{k}$ from $\mathrm{u}_{\mathrm{i}}, 3 \leq \mathrm{i} \leq \alpha$, and there are $\beta-2 \mathrm{k}+2$

vertices of distance more than $\mathrm{k}$ from $\mathrm{u}_{\mathrm{i}}$. Thus, by (1.11)

$C_{n}\left(u_{i}, G^{\prime \prime \prime}, k\right)=\left(\begin{array}{c}\beta-2 k+4 \\ n-1\end{array}\right)-\left(\begin{array}{c}\beta-2 k+2 \\ n-1\end{array}\right)$, for $3 \leq \mathrm{i} \leq \alpha$.

And, for $3 \leq \mathrm{n} \leq \beta-1$ and $2 \leq \mathrm{k} \leq\left\lfloor\frac{\beta-\mathrm{n}+1}{2}\right\rfloor+1$, there are two vertices, namely $\mathrm{v}_{\mathrm{k}}$ and $\mathrm{v}_{\beta-\mathrm{k}+1}$ of distance $\mathrm{k}$ from $\mathrm{u}_{\mathrm{i}}, \mathrm{i}=1,2$, and there are $\beta-2 \mathrm{k}$ vertices of distance more than $\mathrm{k}$ from $\mathrm{u}_{\mathrm{i}}$. Thus, by $(1.11)$

$C_{n}\left(u_{i}, G^{\prime \prime \prime}, k\right)=\left(\begin{array}{c}\beta-2 k+2 \\ n-1\end{array}\right)-\left(\begin{array}{c}\beta-2 k \\ n-1\end{array}\right)$, for $\mathrm{i}=1,2$.

Moreover, if $\mathrm{n}=\beta$ or $\beta+1$, then $\mathrm{k}=1$.

From (5.2.2) and (5.2.3), we get (5.2.1) .

We note that (5.2.1) is not satisfied for $\mathrm{n}=2$, therefore we can obtain $\mathrm{C}_{2}\left(\mathrm{U}, \mathrm{G}^{\prime \prime \prime}, \mathrm{k}\right)$ from Fig. 5.1 , in the next remark:

Remark I: For $n=2, \alpha \geq 3, \beta \geq 4$, then

$\mathrm{C}_{2}\left(\mathrm{U}, \mathrm{G}^{\prime \prime \prime}, \mathrm{k}\right)=2(\alpha-2)+4$, if $2 \leq \mathrm{k} \leq\left\lfloor\frac{\beta-1}{2}\right\rfloor$, 
$\mathrm{C}_{2}\left(\mathrm{U}, \mathrm{G}^{\prime \prime \prime},\left\lfloor\frac{\beta-1}{2}\right\rfloor+1\right)=2(\alpha-2)+2 \mathrm{~h}, \mathrm{C}_{2}\left(\mathrm{U}, \mathrm{G}^{\prime \prime \prime},\left\lfloor\frac{\beta-1}{2}\right\rfloor+2\right)=\mathrm{h}(\alpha-2)$,

where $h=\left[\begin{array}{l}2, \text { if } \beta \text { is even }, \\ 1, \text { if } \beta \text { is odd } .\end{array}\right.$

Remark II: For $\mathrm{n} \geq \beta+2, \mathrm{k} \geq 2, \mathrm{C}_{\mathrm{n}}\left(\mathrm{U}, \mathrm{G}^{\prime \prime \prime}, \mathrm{k}\right)=0$.

Lemma 5.3: For $3 \leq \mathrm{n} \leq \mathrm{p}=\alpha+\beta, \alpha \geq 3, \beta \geq 5$, we have

$\mathrm{C}_{\mathrm{n}}\left(\mathrm{V}, \mathrm{G}^{\prime \prime \prime}, 2\right)=(\beta-2)\left(\begin{array}{l}\mathrm{p}-3 \\ \mathrm{n}-1\end{array}\right)-(\beta-6)\left(\begin{array}{l}\mathrm{p}-5 \\ \mathrm{n}-1\end{array}\right)-2\left[\left(\begin{array}{l}\mathrm{p}-7 \\ \mathrm{n}-1\end{array}\right)+\left(\begin{array}{l}\beta-5 \\ \mathrm{n}-1\end{array}\right)\right]$.

Proof: If $\mathrm{v} \in \mathrm{V}\left(\mathrm{C}_{\beta}\right), \mathrm{S} \subseteq \mathrm{V}\left(\mathrm{G}^{\prime \prime \prime}\right),|\mathrm{S}|=\mathrm{n}-1$, and $\mathrm{d}_{2}(\mathrm{v}, \mathrm{S})=2$ then there are three cases:

Case 1: There are $\alpha$ vertices, namely $\mathrm{u}_{3}, \mathrm{u}_{4}, \ldots, \mathrm{u}_{\alpha}, \mathrm{v}_{3}, \mathrm{v}_{\beta-1}$, of distance 2 from vertex $v_{1}$, and there are $\beta-5$ vertices of distance more than 2 from $v_{1}$. Thus, by (1.11) $\mathrm{C}_{\mathrm{n}}\left(\mathrm{v}_{1}, \mathrm{G}^{\prime \prime \prime}, 2\right)=\left(\begin{array}{l}\mathrm{p}-5 \\ \mathrm{n}-1\end{array}\right)-\left(\begin{array}{l}\beta-5 \\ \mathrm{n}-1\end{array}\right)$.

Since, $C_{n}\left(v_{1}, G^{\prime \prime \prime}, 2\right)=C_{n}\left(v_{\beta}, G^{\prime \prime \prime}, 2\right)$, ( by symmetry $)$, then we have

$C_{n}\left(V_{1}, G^{\prime \prime \prime}, 2\right)=2\left[\left(\begin{array}{c}p-5 \\ n-1\end{array}\right)-\left(\begin{array}{c}\beta-5 \\ n-1\end{array}\right)\right]$, where $V_{1}=\left\{v_{1}, v_{\beta}\right\}$.

Case 2: There are four vertices, namely $\mathrm{u}_{1}, \mathrm{u}_{2}, \mathrm{v}_{4}, \mathrm{v}_{\beta}$, of distance 2 from vertex $\mathrm{v}_{2}$, and there are $p-7$ vertices of distance more than 2 from $v_{2}$. Thus, by (1.11)

$\mathrm{C}_{\mathrm{n}}\left(\mathrm{v}_{2}, \mathrm{G}^{\prime \prime \prime}, 2\right)=\left(\begin{array}{l}\mathrm{p}-3 \\ \mathrm{n}-1\end{array}\right)-\left(\begin{array}{l}\mathrm{p}-7 \\ \mathrm{n}-1\end{array}\right)$.

Since, $C_{n}\left(v_{2}, G^{\prime \prime \prime}, 2\right)=C_{n}\left(v_{\beta-1}, G^{\prime \prime \prime}, 2\right)$, ( by symmetry ), then we have

$\mathrm{C}_{\mathrm{n}}\left(\mathrm{V}_{2}, \mathrm{G}^{\prime \prime \prime}, 2\right)=2\left[\left(\begin{array}{l}\mathrm{p}-3 \\ \mathrm{n}-1\end{array}\right)-\left(\begin{array}{l}\mathrm{p}-7 \\ \mathrm{n}-1\end{array}\right)\right]$, where $\mathrm{V}_{2}=\left\{\mathrm{v}_{2}, \mathrm{v}_{\beta-1}\right\}$.

Case 3: There are two vertices, namely $v_{i-2}, v_{i+2}$, of distance 2 from vertex $v_{i}$, $\mathrm{i}=3,4, \ldots, \beta-2$, and there are $\mathrm{p}-5$ vertices of distance more than 2 from $\mathrm{v}_{\mathrm{i}}$. Thus, by (1.11)

$C_{n}\left(v_{i}, G^{\prime \prime \prime}, k\right)=\left(\begin{array}{l}p-3 \\ n-1\end{array}\right)-\left(\begin{array}{l}p-5 \\ n-1\end{array}\right), 3 \leq i \leq \beta-2$, then

$C_{n}\left(V_{3}, G^{\prime \prime \prime}, k\right)=(\beta-4)\left[\left(\begin{array}{l}p-3 \\ n-1\end{array}\right)-\left(\begin{array}{l}p-5 \\ n-1\end{array}\right)\right], V_{3}=\left\{\begin{array}{lll}v_{i}: i=3,4, & \ldots & , \beta-2\end{array}\right\}$.

Then, from (5.3.2), (5.3.3), and (5.3.4) we get (5.3.1).

From Lemma 5.3, we note that (5.3.1) is satisfied when $\mathrm{n}=2$, that is

$\mathrm{C}_{2}\left(\mathrm{~V}, \mathrm{G}^{\prime \prime \prime}, 2\right)=(\beta-2)(\mathrm{p}-3)-(\beta-6)(\mathrm{p}-5)-2[\mathrm{p}-7+\beta-5]=2(\alpha+\beta)$.

Lemma 5.4: For $3 \leq \mathrm{n} \leq \mathrm{p}=\alpha+\beta, \alpha \geq 3, \beta \geq 7$, and for $3 \leq \mathrm{k} \leq\left\lceil\frac{\beta}{2}\right\rceil-1$, we have 


$$
\begin{array}{r}
\mathrm{C}_{\mathrm{n}}\left(\mathrm{V}, \mathrm{G}^{\prime \prime \prime}, \mathrm{k}\right)=2(\mathrm{k}-2)\left(\begin{array}{c}
\beta-2 \mathrm{k}+1 \\
\mathrm{n}-1
\end{array}\right)-2(\mathrm{k}-1)\left(\begin{array}{c}
\beta-2 \mathrm{k}-1 \\
\mathrm{n}-1
\end{array}\right)-(\beta-2 \mathrm{k}-2)\left(\begin{array}{c}
\mathrm{p}-2 \mathrm{k}-1 \\
\mathrm{n}-1
\end{array}\right) \\
+(\beta-2 \mathrm{k}+2)\left(\begin{array}{c}
\mathrm{p}-2 \mathrm{k}+1 \\
\mathrm{n}-1
\end{array}\right)-2\left(\begin{array}{c}
\mathrm{p}-2 \mathrm{k}-3 \\
\mathrm{n}-1
\end{array}\right) .
\end{array}
$$

Proof: For $3 \leq \mathrm{k} \leq\left\lceil\frac{\beta}{2}\right\rceil-1$, there are four cases for partitioning $\mathrm{V}\left(\mathrm{C}_{\beta}\right)$ corresponding to such values of $\mathrm{k}$.

Case I: There are two vertices, namely $\mathrm{v}_{\mathrm{k}+\mathrm{i}}, \mathrm{v}_{\beta-\mathrm{k}+\mathrm{i}}$, of distance $\mathrm{k}$ from vertex $\mathrm{v}_{\mathrm{i}}$, $\mathrm{i}=1,2, \ldots, \mathrm{k}-2$, and there are $\beta-2 \mathrm{k}-1$ vertices of distance more than $\mathrm{k}$ from $\mathrm{v}_{\mathrm{i}}$. Thus by (1.11)

$\mathrm{C}_{\mathrm{n}}\left(\mathrm{v}_{\mathrm{i}}, \mathrm{G}^{\prime \prime \prime}, \mathrm{k}\right)=\left(\begin{array}{c}\beta-2 \mathrm{k}+1 \\ \mathrm{n}-1\end{array}\right)-\left(\begin{array}{c}\beta-2 \mathrm{k}-1 \\ \mathrm{n}-1\end{array}\right), \mathrm{i}=1,2, \ldots \quad, \mathrm{k}-2$.

Since, $C_{n}\left(v_{i}, G^{\prime \prime \prime}, k\right)=C_{n}\left(v_{\beta-i+1}, G^{\prime \prime \prime}, k\right), i=1,2, \ldots, k-2$ ( by symmetry ), for $2 \leq \mathrm{k} \leq\left\lceil\frac{\beta}{2}\right\rceil-1$, then we have

$\mathrm{C}_{\mathrm{n}}\left(\mathrm{V}_{\mathrm{I}}, \mathrm{G}^{\prime \prime \prime}, \mathrm{k}\right)=2(\mathrm{k}-2)\left[\left(\begin{array}{c}\beta-2 \mathrm{k}+1 \\ \mathrm{n}-1\end{array}\right)-\left(\begin{array}{c}\beta-2 \mathrm{k}-1 \\ \mathrm{n}-1\end{array}\right)\right]$,

$\mathrm{V}_{\mathrm{I}}=\left\{\mathrm{v}_{\mathrm{i}}, \mathrm{v}_{\beta-\mathrm{i}+1}: \mathrm{i}=1,2, \ldots, \mathrm{k}-2\right\}$

Case II: There are $\alpha$ vertices, namely $\mathrm{u}_{3}, \mathrm{u}_{4}, \ldots, \mathrm{u}_{\alpha}, \mathrm{v}_{2 \mathrm{k}-1}, \mathrm{v}_{\beta-1}$, of distance $\mathrm{k}$ from vertex $\mathrm{v}_{\mathrm{k}-1}$, and there are $\beta-2 \mathrm{k}-1$ vertices of distance more than $\mathrm{k}$ from $\mathrm{v}_{\mathrm{k}-1}$. Thus, by $(1.11)$

$\mathrm{C}_{\mathrm{n}}\left(\mathrm{v}_{\mathrm{k}-1}, \mathrm{G}^{\prime \prime \prime}, \mathrm{k}\right)=\left(\begin{array}{c}\mathrm{p}-2 \mathrm{k}-1 \\ \mathrm{n}-1\end{array}\right)-\left(\begin{array}{c}\beta-2 \mathrm{k}-1 \\ \mathrm{n}-1\end{array}\right)$.

Since, $C_{n}\left(v_{k-1}, G^{\prime \prime \prime}, k\right)=C_{n}\left(v_{\beta-k+2}, G^{\prime \prime \prime}, k\right)$, for $2 \leq k \leq\left\lceil\frac{\beta}{2}\right\rceil-1$, we have

$\mathrm{C}_{\mathrm{n}}\left(\mathrm{V}_{\mathrm{II}}, \mathrm{G}^{\prime \prime \prime}, \mathrm{k}\right)=2\left[\left(\begin{array}{c}\mathrm{p}-2 \mathrm{k}-1 \\ \mathrm{n}-1\end{array}\right)-\left(\begin{array}{c}\beta-2 \mathrm{k}-1 \\ \mathrm{n}-1\end{array}\right)\right], \mathrm{V}_{\mathrm{II}}=\left\{\mathrm{v}_{\mathrm{k}-1}, \mathrm{v}_{\beta-\mathrm{k}+2}\right\}$.

Case III: There are four vertices, namely $\mathrm{u}_{1}, \mathrm{u}_{2}, \mathrm{v}_{2 \mathrm{k}}, \mathrm{v}_{\beta}$, of distance $\mathrm{k}$ from vertex $\mathrm{v}_{\mathrm{k}}$, and there are $\mathrm{p}-2 \mathrm{k}-3$ vertices of distance more than $\mathrm{k}$ from $\mathrm{v}_{\mathrm{k}}$. Thus, by (1.11)

$\mathrm{C}_{\mathrm{n}}\left(\mathrm{v}_{\mathrm{k}}, \mathrm{G}^{\prime \prime \prime}, \mathrm{k}\right)=\left(\begin{array}{c}\mathrm{p}-2 \mathrm{k}+1 \\ \mathrm{n}-1\end{array}\right)-\left(\begin{array}{c}\mathrm{p}-2 \mathrm{k}-3 \\ \mathrm{n}-1\end{array}\right)$.

Since, $C_{n}\left(v_{k}, G^{\prime \prime \prime}, k\right)=C_{n}\left(v_{\beta-k+1}, G^{\prime \prime \prime}, k\right)$, for $2 \leq k \leq\left\lceil\frac{\beta}{2}\right\rceil-1$, we have

$\mathrm{C}_{\mathrm{n}}\left(\mathrm{V}_{\text {III }}, \mathrm{G}^{\prime \prime \prime}, \mathrm{k}\right)=2\left[\left(\begin{array}{c}\mathrm{p}-2 \mathrm{k}+1 \\ \mathrm{n}-1\end{array}\right)-\left(\begin{array}{c}\mathrm{p}-2 \mathrm{k}-3 \\ \mathrm{n}-1\end{array}\right)\right], \mathrm{V}_{\mathrm{III}}=\left\{\mathrm{v}_{\mathrm{k}}, \mathrm{v}_{\beta-\mathrm{k}+1}\right\}$.

Case IV: There are two vertices, namely $v_{i-k}, v_{i+k}$, of distance $k$ from vertex $v_{i}$, $\mathrm{i}=\mathrm{k}+1, \mathrm{k}+2, \ldots, \beta-\mathrm{k}$, and there are $\mathrm{p}-2 \mathrm{k}-1$ vertices of distance more than $\mathrm{k}$ from $\mathrm{v}_{\mathrm{i}}$. Thus, by (1.11) 
$\mathrm{C}_{\mathrm{n}}\left(\mathrm{v}_{\mathrm{i}}, \mathrm{G}^{\prime \prime \prime}, \mathrm{k}\right)=\left(\begin{array}{c}\mathrm{p}-2 \mathrm{k}+1 \\ \mathrm{n}-1\end{array}\right)-\left(\begin{array}{c}\mathrm{p}-2 \mathrm{k}-1 \\ \mathrm{n}-1\end{array}\right), \mathrm{k}+1 \leq \mathrm{i} \leq \beta-\mathrm{k}$.

Then,

$\mathrm{C}_{\mathrm{n}}\left(\mathrm{V}_{\mathrm{IV}}, \mathrm{G}^{\prime \prime \prime}, \mathrm{k}\right)=(\beta-2 \mathrm{k})\left[\left(\begin{array}{c}\mathrm{p}-2 \mathrm{k}+1 \\ \mathrm{n}-1\end{array}\right)-\left(\begin{array}{c}\mathrm{p}-2 \mathrm{k}-1 \\ \mathrm{n}-1\end{array}\right)\right]$,

$\mathrm{V}_{\mathrm{IV}}=\left\{\mathrm{v}_{\mathrm{i}}: \mathrm{i}=\mathrm{k}+1, \mathrm{k}+2, \quad \cdots \quad, \beta-\mathrm{k}\right\}$

From (5.4.2) - (5.4.5) we get (5.4.1) .

Also , from Lemma 5.4, we note that (5.4.1) is satisfied when $n=2$, that is

$$
\begin{gathered}
\mathrm{C}_{2}\left(\mathrm{~V}, \mathrm{G}^{\prime \prime \prime}, \mathrm{k}\right)=2(\mathrm{k}-2)(\beta-2 \mathrm{k}+1)-2(\mathrm{k}-1)(\beta-2 \mathrm{k}-1)-(\beta-2 \mathrm{k}-2)(\mathrm{p}-2 \mathrm{k}-1) \\
+(\beta-2 \mathrm{k}+2)(\mathrm{p}-2 \mathrm{k}+1)-2(\mathrm{p}-2 \mathrm{k}-3)=2(\alpha+\beta), \text { for } 3 \leq \mathrm{k} \leq\left\lceil\frac{\beta}{2}\right\rceil-1
\end{gathered}
$$

Lemma 5.5: For $3 \leq \mathrm{n} \leq \mathrm{p}=\alpha+\beta, \alpha \geq 3, \beta \geq 5$, then

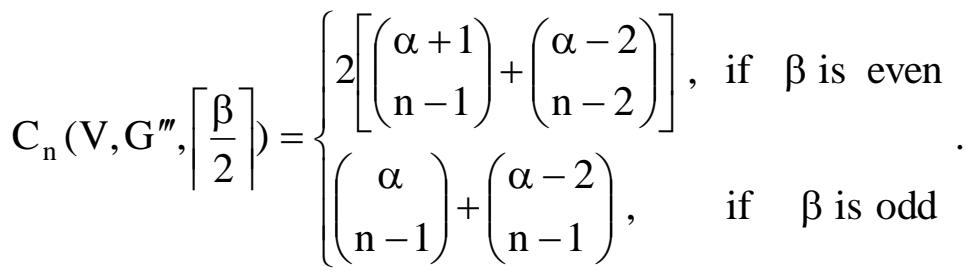

Proof:(i) If $\beta$ is even, then there are $\alpha-1$ vertices, namely $\mathrm{u}_{3}, \mathrm{u}_{4}, \ldots, \mathrm{u}_{\alpha}, \mathrm{v}_{\beta-1}$ of distance $\frac{\beta}{2}$, from vertex $\mathrm{v}_{\frac{\beta}{2}-1}$, and there is no vertex of distance more than $\frac{\beta}{2}$ from $\mathrm{v}_{\frac{\beta}{2}-1}$. Then,

$\mathrm{C}_{\mathrm{n}}\left(\mathrm{v}_{\frac{\beta}{2}-1}, \mathrm{G}^{\prime \prime \prime}, \frac{\beta}{2}\right)=\left(\begin{array}{c}\alpha-1 \\ \mathrm{n}-1\end{array}\right)$,

And there are three vertices, namely $\mathrm{u}_{1}, \mathrm{u}_{2}, \mathrm{v}_{\beta}$, of distance $\frac{\beta}{2}$ from vertex $\mathrm{v}_{\frac{\beta}{2}}$, and there are $\alpha-2$ vertices of distance more than $\frac{\beta}{2}$, from $\mathrm{v}_{\frac{\beta}{2}}$.Then, by (1.11)

$\mathrm{C}_{\mathrm{n}}\left(\mathrm{v}_{\frac{\beta}{2}}, \mathrm{G}^{\prime \prime \prime}, \frac{\beta}{2}\right)=\left(\begin{array}{c}\alpha+1 \\ \mathrm{n}-1\end{array}\right)-\left(\begin{array}{c}\alpha-2 \\ \mathrm{n}-1\end{array}\right)$.

It is clear that $\mathrm{C}_{\mathrm{n}}\left(\mathrm{v}_{\frac{\beta}{2}-\mathrm{r}}, \mathrm{G}^{\prime \prime \prime}, \frac{\beta}{2}\right)=\mathrm{C}_{\mathrm{n}}\left(\mathrm{v}_{\frac{\beta}{2}+\mathrm{r}+1}, \mathrm{G}^{\prime \prime \prime}, \frac{\beta}{2}\right) . \mathrm{r}=0,1$.

Moreover, one may easily check that

$\mathrm{C}_{\mathrm{n}}\left(\mathrm{w}, \mathrm{G}^{\prime \prime \prime}, \frac{\beta}{2}\right)=0$, for $\mathrm{w} \in \mathrm{V}\left(\mathrm{C}_{\beta}\right)-\left\{\mathrm{v}_{\frac{\beta}{2}-1}, \mathrm{v}_{\frac{\beta}{2}}, \mathrm{v}_{\frac{\beta}{2}+1}, \mathrm{v}_{\frac{\beta}{2}+2}\right\}$.

Therefore, from (5.5.1) and (5.5.2) we get 
$\mathrm{C}_{\mathrm{n}}\left(\mathrm{V}, \mathrm{G}^{\prime \prime \prime}, \frac{\beta}{2}\right)=2\left[\left(\begin{array}{l}\alpha+1 \\ \mathrm{n}-1\end{array}\right)+\left(\begin{array}{c}\alpha-2 \\ \mathrm{n}-2\end{array}\right)\right]$.

(ii). If $\beta$ is odd, then there are $\alpha-2$ vertices, namely $\mathrm{u}_{3}, \mathrm{u}_{4}, \ldots, \mathrm{u}_{\alpha}$, of distance $\mathrm{k}=\frac{\beta+1}{2}$ from vertex $\mathrm{v} \in\left\{\mathrm{v}_{\frac{\beta-1}{2}}, \mathrm{v}_{\frac{\beta+3}{2}}\right\}$, and there is no vertex of distance more than $\mathrm{k}=\frac{\beta+1}{2}$ from $\mathrm{v}$. Then,

$\mathrm{C}_{\mathrm{n}}\left(\mathrm{v}, \mathrm{G}^{\prime \prime \prime}, \frac{\beta+1}{2}\right)=\left(\begin{array}{c}\alpha-2 \\ \mathrm{n}-1\end{array}\right), \mathrm{v} \in\left\{\mathrm{v}_{\frac{\beta-1}{2}}, \mathrm{v}_{\frac{\beta+3}{2}}\right\}$.

And, there are two vertices, namely $\mathrm{u}_{1}$ and $\mathrm{u}_{2}$, of distance $\frac{\beta+1}{2}$ from vertex $\mathrm{v}_{\frac{\beta+1}{2}}$, and

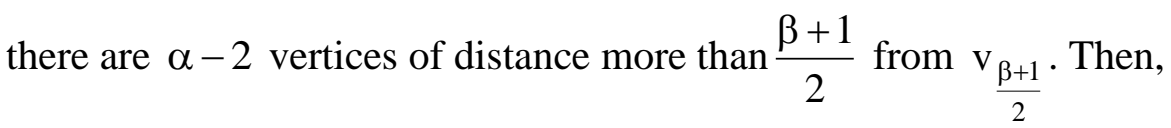

by (1.11)

$\mathrm{C}_{\mathrm{n}}\left(\mathrm{v}_{\frac{\beta+1}{2}}, \mathrm{G}^{\prime \prime \prime}, \frac{\beta+1}{2}\right)=\left(\begin{array}{c}\alpha \\ \mathrm{n}-1\end{array}\right)-\left(\begin{array}{c}\alpha-2 \\ \mathrm{n}-1\end{array}\right)$.

Moreover, $\mathrm{C}_{\mathrm{n}}\left(\mathrm{w}, \mathrm{G}^{\prime \prime \prime}, \frac{\beta+1}{2}\right)=0$, for $\mathrm{w} \in \mathrm{V}\left(\mathrm{C}_{\beta}\right)-\left\{\mathrm{v}_{\frac{\beta-1}{2}}, \mathrm{v}_{\frac{\beta+1}{2}}, \mathrm{v}_{\frac{\beta+3}{2}}\right\}$.

Hence, from (6.5.5), (6.5.6), we get :

$\mathrm{C}_{\mathrm{n}}\left(\mathrm{V}, \mathrm{G}^{\prime \prime \prime}, \frac{\beta+1}{2}\right)=\left(\begin{array}{c}\alpha \\ \mathrm{n}-1\end{array}\right)+\left(\begin{array}{c}\alpha-2 \\ \mathrm{n}-1\end{array}\right)$.

This completes the proof .

From Lemma 5.5 , we get

$\mathrm{C}_{2}\left(\mathrm{~V}, \mathrm{G}^{\prime \prime \prime},\left\lceil\frac{\beta}{2}\right\rceil\right)=\left\{\begin{array}{ll}2(\alpha+2)+\beta-4, & \text { if } \beta \text { is even } \\ 2 \alpha-2, & \text { if } \beta \text { is odd }\end{array}\right.$.

Since, $C_{n}\left(w, G^{\prime \prime \prime}, \frac{\beta}{2}\right)=1$, for $w \in V\left(C_{\beta}\right)-\left\{v_{\frac{\beta}{2}-1}, v_{\frac{\beta}{2}}, v_{\frac{\beta}{2}+1}, v_{\frac{\beta}{2}+2}\right\}$ in (5.5.3) when $n=2$.

Lemma 5.6: For $3 \leq \mathrm{n} \leq \mathrm{p}=\alpha+\beta, \alpha \geq 3, \beta \geq 5$, we have

$C_{n}\left(V, G^{\prime \prime \prime},\left\lceil\frac{\beta}{2}\right\rceil+1\right)=\left[\begin{array}{ll}2\left(\begin{array}{l}\alpha-2 \\ n-1\end{array}\right), & \text { if } \beta \text { is even , } \\ \left(\begin{array}{l}\alpha-2 \\ n-1\end{array}\right), & \text { if } \beta \text { is odd. }\end{array}\right.$

Proof: To simplify the notations, let $\mathrm{m}=\left\lceil\frac{\beta}{2}\right\rceil$.Then, for $\mathrm{k}=\mathrm{m}+1$, there are $\alpha-2$ vertices , namely $\mathrm{u}_{3}, \mathrm{u}_{4}, \ldots, \mathrm{u}_{\alpha}$, of distance $\mathrm{m}+1$ from vertex $\mathrm{v}_{\mathrm{m}}$, and there is no vertex of distance more than $\mathrm{m}+1$

from $\mathrm{v}_{\mathrm{m}}$. Then, 
$\mathrm{C}_{\mathrm{n}}\left(\mathrm{v}_{\mathrm{m}}, \mathrm{G}^{\prime \prime \prime}, \mathrm{m}+1\right)=\left(\begin{array}{c}\alpha-2 \\ \mathrm{n}-1\end{array}\right)$.

Since, $C_{n}\left(v_{m}, G^{\prime \prime \prime}, m+1\right)=C_{n}\left(v_{\beta-m+1}, G^{\prime \prime \prime}, m+1\right)$, this is for even $\beta$ only $\left(\because v_{\beta-m+1} \equiv v_{m+1}\right)$, but for odd $\beta, \mathrm{v}_{\beta-\mathrm{m}+1} \equiv \mathrm{v}_{\mathrm{m}}$

Moreover, $\mathrm{C}_{\mathrm{n}}\left(\mathrm{w}, \mathrm{G}^{\prime \prime \prime}, \mathrm{m}+1\right)=0$, for $\mathrm{w} \in \mathrm{V}\left(\mathrm{C}_{\beta}\right)-\left\{\mathrm{v}_{\mathrm{m}}, \mathrm{v}_{\beta-\mathrm{m}+1}\right\}$.

Hence,

$\mathrm{C}_{\mathrm{n}}\left(\mathrm{V}, \mathrm{G}^{\prime \prime \prime},\left\lceil\frac{\beta}{2}\right\rceil+1\right)=\mathrm{r}^{\prime}\left(\begin{array}{c}\alpha-2 \\ n-1\end{array}\right)$, where $\mathrm{r}^{\prime}=\left[\begin{array}{ll}2 & \text { if } \beta \text { is even } \\ 1 & \text { if } \beta \text { is odd }\end{array}\right.$.

This completes the proof.

From Lemma 5.6, we note that (5.6.1) is satisfied when $\mathrm{n}=2$, that is

$\mathrm{C}_{2}\left(\mathrm{~V}, \mathrm{G}^{\prime \prime \prime},\left\lceil\frac{\beta}{2}\right\rceil+1\right)=\left[\begin{array}{ll}2(\alpha-2), & \text { if } \beta \text { is even, } \\ \alpha-2, & \text { if } \beta \text { is odd. }\end{array}\right.$

Theorem 5.7: For $3 \leq \mathrm{n} \leq \mathrm{p}=\alpha+\beta+\gamma, \alpha \geq 3, \beta, \geq 5$, and $2 \leq \mathrm{k} \leq \delta_{\mathrm{n}}=\operatorname{diam}_{\mathrm{n}} \mathrm{G}^{\prime \prime \prime}$ we have

$$
\begin{aligned}
\mathrm{H}_{\mathrm{n}}\left(\mathrm{G}^{\prime \prime} ; \mathrm{x}\right)= & \mathrm{p}\left(\begin{array}{l}
\mathrm{p}-1 \\
\mathrm{n}-2
\end{array}\right)+\left[\mathrm{p}\left(\begin{array}{l}
\mathrm{p}-1 \\
\mathrm{n}-1
\end{array}\right)-(\alpha-2)\left(\begin{array}{c}
\beta \\
\mathrm{n}-1
\end{array}\right)-2\left(\begin{array}{l}
\beta-2 \\
\mathrm{n}-1
\end{array}\right)-2\left(\begin{array}{l}
\mathrm{p}-5 \\
\mathrm{n}-1
\end{array}\right)-(\beta-2)\left(\begin{array}{l}
\mathrm{p}-3 \\
\mathrm{n}-1
\end{array}\right)\right] \mathrm{x} \\
& +\sum_{k=2}^{\delta_{n}} C_{n}\left(G^{\prime \prime \prime}, k\right) x^{k}
\end{aligned}
$$

and,

$$
\begin{aligned}
\mathrm{W}_{\mathrm{n}}\left(\mathrm{G}^{\prime \prime \prime}\right)= & {\left[\mathrm{p}\left(\begin{array}{l}
\mathrm{p}-1 \\
\mathrm{n}-1
\end{array}\right)-(\alpha-2)\left(\begin{array}{c}
\beta \\
\mathrm{n}-1
\end{array}\right)-2\left(\begin{array}{l}
\beta-2 \\
\mathrm{n}-1
\end{array}\right)-2\left(\begin{array}{l}
\mathrm{p}-5 \\
\mathrm{n}-1
\end{array}\right)-(\beta-2)\left(\begin{array}{l}
\mathrm{p}-3 \\
\mathrm{n}-1
\end{array}\right)\right] } \\
& +\sum_{\mathrm{k}=2}^{\delta_{\mathrm{n}}} \mathrm{kC}_{\mathrm{n}}\left(\mathrm{G}^{\prime \prime \prime}, \mathrm{k}\right)
\end{aligned}
$$

where, $C_{n}\left(G^{\prime \prime \prime}, k\right)=C_{n}\left(U, G^{\prime \prime \prime}, k\right)+C_{n}\left(V, G^{\prime \prime \prime}, k\right)$, for $2 \leq k \leq \delta_{n}$, and $C_{n}\left(U, G^{\prime \prime \prime}, k\right)$, $\mathrm{C}_{\mathrm{n}}\left(\mathrm{V}, \mathrm{G}^{\prime \prime \prime}, \mathrm{k}\right)$ are given in Lemmas 5.2- 5.6 .

Remark III: If $\beta=4, \alpha \geq 3$, then,

$$
\begin{aligned}
& H_{n}\left(G^{\prime \prime \prime} ; x\right)=(\alpha+4)\left(\begin{array}{c}
\alpha+3 \\
n-2
\end{array}\right)+\left[(\alpha+4)\left(\begin{array}{c}
\alpha+3 \\
n-1
\end{array}\right)-(\alpha-2)\left(\begin{array}{c}
4 \\
n-1
\end{array}\right)-2\left(\begin{array}{c}
2 \\
n-1
\end{array}\right)-2\left(\begin{array}{l}
\alpha-1 \\
n-1
\end{array}\right)-2\left(\begin{array}{l}
\alpha+1 \\
n-1
\end{array}\right)\right] x \\
& +\left[2\left(\begin{array}{l}
\alpha+1 \\
n-1
\end{array}\right)+(\alpha-2)\left(\begin{array}{c}
4 \\
n-1
\end{array}\right)-(\alpha-4)\left(\begin{array}{c}
2 \\
n-1
\end{array}\right)+2\left(\begin{array}{l}
\alpha-2 \\
n-2
\end{array}\right)\right] x^{2}+\left[(\alpha-2)\left(\begin{array}{c}
2 \\
n-1
\end{array}\right)+2\left(\begin{array}{l}
\alpha-2 \\
n-1
\end{array}\right)\right] x^{3} \text {. }
\end{aligned}
$$

Remark IV: From (5.3.5) , (5.4.6), (5.5.7) and (5.6.2), we get

$\mathrm{C}_{2}\left(\mathrm{~V}, \mathrm{G}^{\prime \prime \prime}, \mathrm{k}\right)=2(\alpha+\beta), \quad$ if $2 \leq \mathrm{k} \leq\left\lceil\frac{\beta}{2}\right\rceil-1$

$\mathrm{C}_{2}\left(\mathrm{~V}, \mathrm{G}^{\prime \prime \prime},\left\lceil\frac{\beta}{2}\right\rceil\right)= \begin{cases}2(\alpha+2)+\beta-4, & \text { if } \beta \text { is even } \\ 2 \alpha-2, & \text { if } \beta \text { is odd }\end{cases}$ 
$\mathrm{C}_{2}\left(\mathrm{~V}, \mathrm{G}^{\prime \prime \prime},\left\lceil\frac{\beta}{2}\right\rceil+1\right)=\left[\begin{array}{ll}2(\alpha-2), & \text { if } \beta \text { is even }, \\ \alpha-2, & \text { if } \beta \text { is odd }\end{array}\right.$

Since, $C\left(G^{\prime \prime \prime}, k\right)=\frac{1}{2}\left[C_{2}\left(U, G^{\prime \prime \prime}, k\right)+C_{2}\left(V, G^{\prime \prime \prime}, k\right)\right]$, for $2 \leq k \leq\left\lceil\frac{\beta}{2}\right\rceil+1$, where, $\mathrm{C}_{2}\left(\mathrm{U}, \mathrm{G}^{\prime \prime \prime}, \mathrm{k}\right)$ and $\mathrm{C}_{2}\left(\mathrm{~V}, \mathrm{G}^{\prime \prime \prime}, \mathrm{k}\right)$ are given in Remarks II and IV, we get the next corollary.

Corollary 5.8: For, $\alpha \geq 3, \beta \geq 5$, the Hosoya polynomial of $G^{\prime \prime}$ of order $p=\alpha+\beta$ is given by:

$$
\begin{aligned}
\mathrm{H}\left(\mathrm{G}^{\prime \prime \prime} ; \mathrm{x}\right)=(\alpha+\beta)+\frac{1}{2}[\alpha(\alpha-1)+ & 2 \beta+8)] \mathrm{x}+(2 \alpha+\beta) \sum_{\mathrm{k}=2}^{\lceil\beta / 2\rceil-1} \mathrm{x}^{\mathrm{k}} \\
& +\left\{\begin{array}{l}
(2 \alpha+\beta / 2) \mathrm{x}^{\beta / 2}+2(\alpha-2) \mathrm{x}^{\beta / 2+1}, \text { if } \beta \text { is even } \\
2(\alpha-1) \mathrm{x}^{(\beta+1) / 2}+(\alpha-2) \mathrm{x}^{(\beta+1) / 2+1}, \text { if } \beta \text { is odd }
\end{array} .\right.
\end{aligned}
$$

And, Wiener index of $\mathrm{G}^{\prime \prime \prime}$ is

$\mathrm{W}\left(\mathrm{G}^{\prime \prime \prime}\right)=\frac{1}{2} \alpha(\alpha-1)+\frac{\beta}{2}(3 \alpha-4)+\frac{\beta^{2}}{4}\left(\alpha+\frac{\beta}{2}\right)$, if $\beta$ is even,

$\mathrm{W}\left(\mathrm{G}^{\prime \prime \prime}\right)=\frac{1}{2} \alpha\left(\alpha-\frac{1}{2}\right)+\frac{\beta}{2}\left(3 \alpha-\frac{17}{4}\right)+\frac{\beta^{2}}{4}\left(\alpha+\frac{\beta}{2}\right)$, if $\beta$ is odd .

Remark III: If $\beta=4, \alpha \geq 3$, then,

- $\mathrm{H}\left(\mathrm{G}^{\prime \prime \prime} ; \mathrm{x}\right)=(\alpha+4)+\frac{1}{2}[\alpha(\alpha-1)+16] \mathrm{x}+(2 \alpha+2) \mathrm{x}^{2}+2(\alpha-2) \mathrm{x}^{3}$,

- $\mathrm{W}\left(\mathrm{G}^{\prime \prime \prime}\right)=\frac{1}{2} \alpha(\alpha+19)$. 


\section{REFERENCES}

[1] Abdullah, H. O. (2007), " Hosoya polynomials of Steiner Distance of Some Graphs", Ph.D. Thesis, University of Salahaddin \Erbil, Erbil, Iraq.

[2] Ahmed, H. G. (2007), "On Wiener Polynomials of n-Distance in Graphs", M.Sc. Thesis, University of Dohuk, Dohuk, Iraq.

[3] Ali , A.A. and Ali , A.M. ; (2006). "Wiener Polynomials of the Generalized Distance for some special Graphs", Raf. J. Comp. Sc. And Maths. Vol.3 , No.2 , pp.103-120.

[4] Aziz, A.S. (2006), "The Width Distance and w-Wiener Polynomials of a Graph", M.Sc. Thesis, University of Mosul, Mosul, Iraq.

[5] Buckley , F. and Harary , F.; (1990). Distance in Graphs. Addison -Wesley, Redwood, California .

[6] Chartrand G. and Lesniak , L.; (1986). Graphs and Digraphs, $2^{\text {nd }} e d$., Wadsworth and Brooks / Cole, California .

[7] Gutman, I. , (1993). " Some properties of Wiener polynomial "; Graph Theory Notes of New York; XXV. The New York Academy of Siences, pp. 13-17.

[8] Saeed , W.A.M. ; (1999) . “Wiener Polynomials of Graphs “ , Ph.D. thesis, Mosul University, Mosul. 